\title{
A molecular theory for two-photon and three-photon fluorescence polarization
}

\author{
J. M. Leeder and D. L. Andrews ${ }^{\mathrm{a})}$ \\ School of Chemistry, University of East Anglia, Norwich NR4 7TJ, United Kingdom
}

(Received 9 November 2010; accepted 31 January 2011; published online 2 March 2011)

\begin{abstract}
In the analysis of molecular structure and local order in heterogeneous samples, multiphoton excitation of fluorescence affords chemically specific information and high-resolution imaging. This report presents the results of an investigation that secures a detailed theoretical representation of the fluorescence polarization produced by one-, two-, and three-photon excitations, with orientational averaging procedures being deployed to deliver the fully disordered limits. The equations determining multiphoton fluorescence response prove to be expressible in a relatively simple, generic form, and graphs exhibit the functional form of the multiphoton fluorescence polarization. Amongst other features, the results lead to the identification of a condition under which the fluorescence produced through the concerted absorption of any number of photons becomes completely unpolarized. It is also shown that the angular variation of fluorescence intensities is reliable indicator of orientational disorder. () 2011 American Institute of Physics. [doi:10.1063/1.3556537]
\end{abstract}

\section{INTRODUCTION}

In laser-based studies of fluorescence, it is well known that polarization features of the emission convey rich information on structural details of the sample, particularly in condensed phase molecular media. The character of emission from each fluorescent species owes its origin to both the properties of the input light and the internal configuration of transitions and energy levels. Polarization-resolved measurements afford key information on molecular structure, and the degree or extent of local orientational order. ${ }^{1,2}$ The elucidation of such information is widely exploited in fluorescence imaging, where the objective to secure quality, three-dimensionally resolved images is supplemented by a scope to accurately distinguish the location, concentration and structure of specific chromophores. ${ }^{3-5}$ (In this paper the term "chromophore" is used to signify a molecular component or label that can absorb both the light and fluoresce-the term "fluorophore" being equally valid.) In connection with conventional (single photon) fluorescence, such principles are well known and widely applied across a diverse range of physical systems. Numerous studies have focused on confined, highly ordered materials where chromophores are held in crystalline structures, ${ }^{6-8}$ or samples, such as cell membranes, molecular films or fiber, where they are less rigidly bound to a physical matrix. ${ }^{9,10}$ In such instances, the rotational freedom of the targeted species is commonly restricted, enforcing a degree of orientational order relative to the external structure. Whereas polarization-derived information is often restricted to two spatial dimensions, the determination of three-dimensional orientation can also be explored. ${ }^{11}$ Numerous investigations have extended the scope of such studies into the single-molecule regime, to elucidate information that is obscured in ensemble studies. ${ }^{12-14}$

\footnotetext{
a) Author to whom correspondence should be addressed. Electronic mail: d.1.andrews@uea.ac.uk.
}

An ever-increasing number of studies exploit the advantages inherent in multiphoton excitation, such as the identification of "dark states," 15 photoselection methods, ${ }^{16-20}$ and studies on the effects of molecular orientational diffusion $^{21-23}$-as well as the opportunities for chemically specific imaging with unprecedented resolution. For simplicity, such experiments are usually performed with a single laser beam, although it has been recognized that the use of two or even three beams with different optical frequencies can offer additional tiers of information. ${ }^{24-27} \mathrm{Ex}-$ perimental applications are particularly prevalent in biological studies, where they afford a capability for imaging to subcellular resolution, with limited photo-damage, and without any need to suppress light scattering. ${ }^{28-32}$ One of the most appealing features of multiphoton-induced imaging is its adaptability, and the fact that the associated instrumentation is also often well suited to additional means of sample interrogation. Commonly used, complementary modes of measurement include second-harmonic generation, sumfrequency generation, coherent anti-Stokes Raman scattering, and Raman spectroscopy-all are frequently combined with multiphoton fluorescence. ${ }^{33,34}$ While three-photon microscopy in particular has been less commonly studied than its two-photon counterpart, it is recognized that image contrast can be enhanced as the number of concerted photon interactions increases. ${ }^{35,36}$ The incorporation of additional techniques can permit the visual sectioning of specific molecular domains within bulk material, expanding the potential for applying multiphoton imaging as a tool in structural diagnostics. Nonetheless, securing all of the orientational information that is latent in multiphoton fluorescence is technically demanding, and at present it is compromised by the lack of a complete understanding - certainly in the case of three-photon excitation studies - of how the polarization response from a fully disordered system relates to the detailed electronic properties of the constituent chromophores. 
This report presents the results of an investigation aiming to secure a robust, thorough and comprehensive representation of the fluorescence polarization properties generated in response to one-, two- and three-photon excitation of molecular chromophores. The single-photon case is included both as a means of introducing the theoretical formalism, and to help elicit patterns of response between the different orders, established subsequently. Although two-photon studies are more common, the selection rules for three-photon excitation offer the possibility of access to states that are not amenable to one- or two-photon excitation..$^{24,27,37-41}$ Results established by means of an isotropic orientational average determine the induced fluorescence response generated within a fully disordered molecular environment-a complete system, or microdomains within a complete system, in which all chromophores are randomly oriented in three dimensions. It can be anticipated that the averaged results will prove their value in determining the random orientation limit of a dynamic spectrum, providing a means by which multiphoton imaging can be further developed to monitor and quantify variations in chromophore orientation. In a system with some orientational order (for example one that is undergoing a chemical or biological function, or responding to a controlled external stimulus), the extent of deviation in the fluorescence response, compared to that expected from an isotropic sample, will quantifiably register the degree of the order.

In Sec. II, essential details relating to the theoretical representation of one-, two-, and three-photon induced fluorescence are established, casting the output signals in terms of their associated electric polarization and molecular transition moment properties through standard methods of quantum electrodynamics (QED), as described in numerous texts and reviews. ${ }^{42-46}$ In Sec. III, orientational averaging procedures are detailed, the results of which define the fully disordered limits of both the single and multiphoton fluorescence processes. The analysis requires implementation of fourth-, sixth-, and eighth-rank tensor averages, ${ }^{47}$ of which only the former two are widely documented; nonetheless their calculational principles have been deployed across a wide range of photophysical processes, recently including coupled systems and interactions such as quantum dot assemblies, ${ }^{48,49}$ van der Waals dispersion energies and Casimir effects, ${ }^{50,51}$ and the evolution of excitons in conjugated polymers and chromophore aggregates. ${ }^{52,53}$ However, the complexity of the averaging procedure escalates rapidly with the tensor rank, and it is not surprising that eighth rank averaging has rarely been utilized-until recently, in the context of laser-controlled fluorescence. ${ }^{54}$ The significance, patterns and applications of these results are discussed in Sec. IV.

\section{MULTIPHOTON FLUORESCENCE THEORY}

To approach the key polarization issues, it is appropriate to begin with a representation of the optical process in its entirety, subsuming the single- or multi-photon absorption of laser input, and the emission of fluorescent radiation. Each stage occurs with an efficiency that is determined by the strength of coupling between the ground and relevant excited electronic levels, dependent on the component values of the relevant transition dipoles or multiphoton tensors. A detailed theoretical representation is built on the basis of parameters delivered by quantum theory-specifically the quantum amplitudes (or "matrix elements," as they are in principle derivable for any specified pair of states) for the initial excitation and for the fluorescent decay. The process efficiency, determined by Fermi's rule, is in each case proportional to the modulus square of the matrix element: ${ }^{55}$ the excitation and emission events are mutually independent and in practice they occur in a stepwise fashion. For our purposes we shall assume the validity of a Born-Oppenheimer separation of wavefunctions and focus upon electronic transitions; the corresponding vibrational energies are generally small compared to the electronic state energy differences. Also we assume a development through molecular states that is, typically, associated with electric dipole transitions; the contribution from both magnetic and higher order electric contributions is generally insignificant. The theory that follows will provide a means for interrogating the extent of correlation between the transition moments associated with absorption and emission. Specific attention will be given to the extent to which fluorescence retains a directionality of polarization from the initial excitation.

To achieve fluorescence intensity results amenable to experimental application, matrix elements of the form $M_{f i}(\xi)$ are defined for each electronic transition coupling a specified initial state, $i$, and final state, $f$, in a chromophore $\xi$. The output fluorescence signal, $I_{\mathrm{flu}}^{(n)}(\phi)$, is a function of the experimentally controllable angle between the polarization vector of the incident light and the resolved polarization of the emission, $\phi$, and it can be cast in general terms of the separate matrix elements for $n$th order multiphoton absorption and single-photon emission, namely $M_{\nu 0}^{(n)}(\xi)$ and $M_{0 \alpha}(\xi)$, respectively. Our representation allows the possibility for excited state processes such as internal conversion, hindered rotation, rotational diffusion, intramolecular energy transfer, etc., to intervene between the excitation and radiative decay. Adopting labels 0 and $v$ to denote the molecular ground and initially excited energy levels, and $\alpha$ for the level from which emission occurs, the intensity of fluorescence can be cast as follows:

$$
I_{f l u}^{(n)}(\phi)=K^{(n)} \sum_{\xi}\left\langle\left|M_{\nu 0}^{(n)}(\xi)\right|^{2}\left|M_{0 \alpha}(\xi)\right|^{2}\right\rangle .
$$

The fluorescence signal in Eq. (1) is thus portrayed in terms of the physically separable efficiencies of the absorption and emission processes; the constant of proportionality $K^{(n)}$ is itself dependent on experimental parameters including the $n$th power of the mean laser irradiance, and the degree of $n$th order coherence. ${ }^{56}$ To assess the relationship between $I_{\text {flu }}^{(n)}$ and $\phi$ for a fully disordered system in which molecular chromophores (more specifically, the transition moments associated with multiphoton absorption and single photon emission) are randomly oriented relative to the input propagation, the angular brackets in Eq. (1) are implemented in terms of an orientational average. First, to determine the results for one-, two-, and three-photon induced fluorescence, it is necessary to define the form of all associated matrix elements. Each is derived by standard methods; the underlying principles are introduced in a detailed description of single-photon induced fluorescence that follows. 


\section{A. One-photon induced fluorescence}

It is expedient to concisely review the simplest, familiar case of one-photon induced fluorescence, as it establishes the methods to be used for the more intricate multiphoton cases that follow. Theory for the process of single-photon induced fluorescence is characterized by the development of two distinct matter-radiation interactions. The first describes the optical excitation of a chromophore by single-photon absorption, inducing an electronic transition from the ground to an accessible excited state configuration. The second interaction entails molecular relaxation and photon emission, usually returning the chromophore to its ground electronic state. The associated matrix elements for each interaction determine the overall quantum probability of both the molecular and radiation states developing from a given initial state $|I\rangle$ of the system (molecule + radiation) to a specified final state $|F\rangle$, progressing through real, physically identifiable intermediate states, $|R\rangle$ and $\left|R^{\prime}\right\rangle$ - the latter allowed to differ by accommodating any ultrafast intramolecular redistribution processes that might precede emission, such relaxation typically manifest in a Stokes shift. For the complete process of one-photon induced fluorescence, the initial, intermediate, and final system states are thus described as

$$
\left.\begin{array}{rl}
|I\rangle & =\left|\xi_{0} ; q(\mathbf{p}, \lambda)\right\rangle, \\
|R\rangle & =\left|\xi_{v} ;(q-1)(\mathbf{p}, \lambda)\right\rangle, \\
\left|R^{\prime}\right\rangle & =\left|\xi_{\alpha} ; 0\left(\mathbf{p}^{\prime}, \lambda^{\prime}\right)\right\rangle, \\
|F\rangle & =\left|\xi_{0} ; 1\left(\mathbf{p}^{\prime}, \lambda^{\prime}\right)\right\rangle,
\end{array}\right\}
$$

utilizing $\mathbf{p}$ and $\lambda$ to respectively represent the wave-vectors and polarizations of the input beam, distinct from $\mathbf{p}^{\prime}$ and $\lambda^{\prime}$, properties of the output fluorescence. The input mode conveys $q$ photons within a quantization volume that encloses the absorbing chromophore. For simplicity the state of the optical output mode is omitted from the state descriptions of $|I\rangle$ and $|R\rangle$ because that mode suffers no change in the intervening (absorption) transition; equally the state of the input beam, thereafter unchanged, is omitted from the designations of $\left|R^{\prime}\right\rangle$ and $|F\rangle$ as their coupling only concerns the fluorescence output.

\section{Initial absorption}

The initial photon absorption drives evolution between the system states $|I\rangle$ and $|R\rangle$ of Eq. (2), the photon promoting an electronic transition between molecular states $\xi_{0}$ and $\xi_{v}$. The required matrix element for single photon absorption emerges as

$$
M_{\nu 0}^{(1)}(\xi)=\left\langle\xi_{\nu} ;(q-1)(\mathbf{p}, \lambda)\left|H_{\text {int }}(\xi)\right| \xi_{0} ; q(\mathbf{p}, \lambda)\right\rangle,
$$

in which the molecular and radiation system states are coupled through the interaction Hamiltonian, $H_{\text {int }}(\xi)$. The latter is expressible in the following form, where $\mu_{i}(\xi)$ is a component of the electric dipole operator for chromophore $\xi$;

$$
H_{\text {int }}(\xi)=-\varepsilon_{0}^{-1} \mu_{i}(\xi) \cdot d_{i}^{\perp}\left(\mathbf{R}_{\xi}\right),
$$

using the convention of summation over repeated Cartesian subscripts. The transverse electric displacement field operator
$d_{i}^{\perp}\left(\mathbf{R}_{\xi}\right)$ at position $\mathbf{R}_{\xi}$ acts upon the system radiation states within the volume $V$ as

$$
\begin{aligned}
d_{i}^{\perp}\left(\mathbf{R}_{\xi}\right)= & \sum_{\boldsymbol{p}, \lambda}\left(\frac{\hbar c p \varepsilon_{0}}{2 V}\right)^{1 / 2} i\left[e_{i}^{(\lambda)}(\mathbf{p}) a^{(\lambda)}(\mathbf{p}) \exp \left(i \mathbf{p} . \mathbf{R}_{\xi}\right)\right. \\
& \left.-\bar{e}_{i}^{(\lambda)}(\mathbf{p}) a^{\dagger(\lambda)}(\mathbf{p}) \exp \left(-i \mathbf{p} . \mathbf{R}_{\xi}\right)\right],
\end{aligned}
$$

where $e_{i}^{(\lambda)}$ is the unit electric polarization vector with complex conjugate $\bar{e}_{j}^{(\lambda)}$. The electric field operator is linear in both $a$ and $a^{\dagger}$, which are the optical mode annihilation and creation operators respectively; hence each operation of $d_{i}^{\perp}\left(\mathbf{R}_{\xi}\right)$ is responsible for either the creation or annihilation of a single photon. Substitution of Eq. (4) into Eq. (3) deploys the photon annihilation operator in Eq. (5), the resulting matrix element for one-photon absorption is as follows:

$$
M_{\nu 0}^{(1)}(\xi)=-i\left(\frac{q \hbar c p}{2 \varepsilon_{0} V}\right)^{1 / 2} e_{i}^{(\lambda)} \mu_{i}^{\nu 0} \exp \left(i \mathbf{p} \cdot \mathbf{R}_{\xi}\right)
$$

\section{Single-photon emission}

The emission engages electronic decay of the excited chromophore and creation of a single photon into the vacuum radiation field, the process expressed by Eq. (2) as a transition between system states $\left|R^{\prime}\right\rangle$ and $|F\rangle$. The matrix element now engages the photon creation operator in Eq. (5), giving

$$
M_{0 \alpha}(\xi)=i\left(\frac{\hbar c p^{\prime}}{2 \varepsilon_{0} V}\right)^{1 / 2} \bar{e}_{i}^{\left(\lambda^{\prime}\right)} \mu_{i}^{0 \alpha} \exp \left(-i \mathbf{p}^{\prime} \cdot \mathbf{R}_{\xi}\right)
$$

On substitution of the derived matrix elements for both absorption and emission into Eq. (1), a complete expression for the signal output following single photon excitation emerges:

$$
I_{\mathrm{flu}}^{(1)}(\phi)=K^{(1)} \sum_{\xi}\left\langle\left(e_{i}^{(\lambda)} \bar{e}_{j}^{\left(\lambda^{\prime}\right)} \bar{e}_{k}^{(\lambda)} e_{l}^{\left(\lambda^{\prime}\right)} \mu_{i}^{\nu 0} \mu_{j}^{0 \alpha} \bar{\mu}_{k}^{\nu 0} \bar{\mu}_{l}^{0 \alpha}\right)\right\rangle,
$$

where the modulus squares of Eqs. (6) and (7) have been employed and the product of parameters within the parentheses of each matrix element is incorporated into the proportionality constant $K^{(1)}$. For convenience in the orientational averaging procedure to be utilized in Sec. III, a new notation is now introduced in which the products of the unit electric polarization vectors, and those of the molecular transition moments, are each incorporated into second rank tensors as follows:

$$
I_{\mathrm{flu}}^{(1)}(\phi)=\sum_{\xi} K^{(1)}\left\langle S_{i j} \bar{S}_{k l} T_{i j} \bar{T}_{k l}\right\rangle \text {, }
$$

where specifically $S_{i j}$ and $\bar{S}_{i j}$ denote $e_{i}^{(\lambda)} \bar{e}_{j}^{\left(\lambda^{\prime}\right)}$ and $\bar{e}_{i}^{(\lambda)} e_{j}^{\left(\lambda^{\prime}\right)}$. Likewise, the molecular transition moment products described by $T_{i j}$ and $\bar{T}_{i j}$ correspond to $\mu_{i}^{\nu 0} \mu_{j}^{0 \alpha}$ and $\bar{\mu}_{i}^{\nu 0} \bar{\mu}_{j}^{0 \alpha}$. In these examples, and in all subsequent applications of this notation, the last index in the electric polarization and molecular transition tensors relates to photon emission. Equation (9) thus expresses a result that embraces, in the term within angular brackets, the angular disposition of the chromophore transition moments with respect to the input and 
output polarization vectors. In a rigidly oriented system, by forgoing the orientational average the result would thus exhibit a dependence on $\cos ^{2} \eta \cos ^{2} \theta$, where $\eta$ is the angle between the absorption moment and the input polarization and $\theta$ is that between the emission moment and the fluorescence polarization.

\section{B. Two-photon induced fluorescence}

Two-photon induced fluorescence is characterized by the development of three distinct matter-radiation interactionsthe concerted absorption of two photons followed by onephoton emission. The initial, intermediate and final system states are defined as

$$
\begin{aligned}
|I\rangle & =\left|\xi_{0} ; q(\mathbf{p}, \lambda)\right\rangle \\
|R\rangle & =\left|\xi_{v} ;(q-2)(\mathbf{p}, \lambda)\right\rangle, \\
\left|R^{\prime}\right\rangle & =\left|\xi_{\alpha} ; 0\left(\mathbf{p}^{\prime}, \lambda^{\prime}\right)\right\rangle \\
|F\rangle & =\left|\xi_{0} ; 1\left(\mathbf{p}^{\prime}, \lambda^{\prime}\right)\right\rangle
\end{aligned}
$$

First, we focus on the matrix element for the two-photon transition between $|I\rangle$ and $|R\rangle$.

\section{Two-photon absorption}

The acquisition of two photon energies by the chromophore, in its excitation to level $\xi_{v}$, leads to a system state $|R\rangle$. The associated matrix element entails a progression through an intermediate system state $|A\rangle$, in which one photon has been annihilated and the chromophore, lacking a resonant level to match the photon energy, is accordingly in a superposition of virtual molecular states $\left|\xi_{r}\right\rangle$ :

$$
\left.\begin{array}{rl}
|I\rangle & =\left|\xi_{0} ; q(\mathbf{p}, \lambda)\right\rangle, \\
|A\rangle & =\left|\xi_{r} ;(q-1)(\mathbf{p}, \lambda)\right\rangle, \\
|R\rangle & =\left|\xi_{v} ;(q-2)(\mathbf{p}, \lambda)\right\rangle .
\end{array}\right\}
$$

Any energy nonconserving state $|A\rangle$ can be sustained as long as it is allowed by the time-energy uncertainty principle - and this is reflected in a weighting factor, varying with the inverse of the mismatch energy, in the following expression for the second-order matrix element:

$$
M_{\nu 0}^{(2)}(\xi)=\sum_{r} \frac{\left\langle\xi_{v} ;(q-2)(\mathbf{p}, \lambda)\left|H_{\mathrm{int}}(\xi)\right| \xi_{r} ;(q-1)(\mathbf{p}, \lambda)\right\rangle\left\langle\xi_{r} ;(q-1)(\mathbf{p}, \lambda)\left|H_{\mathrm{int}}(\xi)\right| \xi_{0} ; q(\mathbf{p}, \lambda)\right\rangle}{\left(E_{0}+2 \hbar c p\right)-\left(E_{r}+\hbar c p\right)}
$$

A necessary summation is made over all possible intermediate states, as required by quantum principles. The matrix element is developed by substitution of Eqs. (4) and (5) into Eq. (12) such that

$$
\begin{aligned}
M_{\nu 0}^{(2)}(\xi)= & q_{2}^{1 / 2}\left(\frac{\hbar c p}{2 \varepsilon_{0} V}\right) e_{i}^{(\lambda)} e_{j}^{(\lambda)} \frac{1}{2} \sum_{r}\left(E_{0 r}+\hbar c p\right)^{-1} \\
& \times\left(\mu_{i}^{v r} \mu_{j}^{r 0}+\mu_{j}^{v r} \mu_{i}^{r 0}\right)
\end{aligned}
$$

Here, the quantization volume initially contains the chromophore and two photons of the incident radiation; the factor of $q_{2}^{1 / 2} \equiv[q(q-1)]^{1 / 2}$ correspondingly arises from the successive operations of the photon annihilation operator. The above expression exploits the symmetry of the electric polarization terms $e_{i}^{(\lambda)} e_{j}^{(\lambda)}$ with respect to exchange of the indices $i$ and $j$. The two dipole product contributions in Eq. (13) relate to each of the possible time-orderings in which the two, indistinguishable input photons can be annihilated; the factor of $1 / 2$ is introduced to preclude over-counting. The above two-photon absorption matrix element can thus be presented as

$$
M_{\nu 0}^{(2)}(\xi)=\left(\frac{q_{2}^{1 / 2} \hbar c p}{2 \varepsilon_{0} V}\right) e_{i}^{(\lambda)} e_{j}^{(\lambda)} \alpha_{(i j)}^{\nu 0}
$$

where, in the above expression, bracketed subscripts denote symmetry in the enclosed indices. The second rank molecular response tensor $\alpha_{(i j)}^{\nu 0}$ is defined thus:

$$
\alpha_{(i j)}^{\nu 0}=\frac{1}{2} \sum_{r}\left(E_{0 r}+\hbar c p\right)^{-1}\left(\mu_{i}^{v r} \mu_{j}^{r 0}+\mu_{j}^{v r} \mu_{i}^{r 0}\right)
$$

Meath and Power demonstrated the importance of including in this expression static dipole moments (which arise in the state summands for $r=0$ or $v$ ), in the case of polar chromophores. $^{57}$

\section{Full process}

Returning to Eq. (7), the matrix element for one-photon emission is now deployed, and substitution of this and Eq. (14) into Eq. (1) determines the two-photon induced fluorescence signal. In the following expression the proportionality constant $K^{(2)}$ contains a factor $q_{2}$ which, in general, conveys a quadratic dependence on the intensity, and which is also a function of the photon statistics of the input beam. Specifically, $q_{2} \Rightarrow g^{(2)} \bar{q}^{2}$, where $\bar{q}$ is the mean number of input photons in the quantization volume, and $g^{(2)}$ is the degree of second order coherence. ${ }^{56}$ Thus we have

$$
I_{\mathrm{flu}}^{(2)}(\phi)=\sum_{\xi} K^{(2)}\left\langle S_{(i j) k} \bar{S}_{(l m) n} T_{(i j) k} \bar{T}_{(l m) n}\right\rangle,
$$


here expressing the electric vector and molecular transition moment products as third rank tensors such that $S_{(i j) k}$ and $\bar{S}_{(i j) k}$ correspond to $e_{i}^{(\lambda)} e_{j}^{(\lambda)} \bar{e}_{k}^{\left(\lambda^{\prime}\right)}$ and $\bar{e}_{i}^{(\lambda)} \bar{e}_{j}^{(\lambda)} e_{k}^{\left(\lambda^{\prime}\right)}$, while $T_{(i j) k}$ and $\bar{T}_{(i j) k}$ signify $\alpha_{(i j)}^{\nu 0} \mu_{k}^{0 \alpha}$ and $\bar{\alpha}_{(i j)}^{\nu 0} \bar{\mu}_{k}^{0 \alpha}$, respectively. In this case, for an oriented sample, the dependence on emission angle is again $\cos ^{2} \theta$. However the dependence on input polarization is considerably more intricate, being determined by a weighted combination of $\cos ^{2}$ functions for each angle between the input polarization vector and one of a bundle of transition moments, i.e., $\boldsymbol{\mu}^{\nu r}, \boldsymbol{\mu}^{r 0}$, for each level $r$.

\section{Three-photon induced fluorescence}

For three-photon induced fluorescence, the initial, intermediate excited and final system states are

$$
\begin{aligned}
|I\rangle & =\left|\xi_{0} ; q(\mathbf{p}, \lambda)\right\rangle, \\
|R\rangle & =\left|\xi_{v} ;(q-3)(\mathbf{p}, \lambda)\right\rangle, \\
\left|R^{\prime}\right\rangle & =\left|\xi_{\alpha} ; 0\left(\mathbf{p}^{\prime}, \lambda^{\prime}\right)\right\rangle, \\
|F\rangle & =\left|\xi_{0} ; 1\left(\mathbf{p}^{\prime}, \lambda^{\prime}\right)\right\rangle .
\end{aligned}
$$

There are four photon-matter interactions-one of which is one-photon emission, again characterized by Eq. (7).

\section{Three-photon absorption}

For the three-photon transition between $|I\rangle$ and $|R\rangle$ there are two distinct virtual intermediate system states, $|A\rangle$ and $|B\rangle$ as follows:

$$
\left.\begin{array}{rl}
|I\rangle & =\left|\xi_{0} ; q(\mathbf{p}, \lambda)\right\rangle, \\
|A\rangle & =\left|\xi_{r} ;(q-1)(\mathbf{p}, \lambda)\right\rangle, \\
|B\rangle & =\left|\xi_{s} ;(q-2)(\mathbf{p}, \lambda)\right\rangle, \\
|R\rangle & =\left|\xi_{v} ;(q-3)(\mathbf{p}, \lambda)\right\rangle .
\end{array}\right\}
$$

The three-photon matrix element emerges as

$$
\begin{aligned}
M_{v 0}^{(3)}(\xi)= & \sum_{r, s}\left(\left(E_{0 r}+\hbar c p\right)\left(E_{0 s}+2 \hbar c p\right)\right)^{-1} \\
& \times\left\langle\xi_{v} ;(q-3)(\mathbf{p}, \lambda)\left|H_{\mathrm{int}}(\xi)\right| \xi_{s} ;(q-2)(\mathbf{p}, \lambda)\right\rangle \\
& \times\left\langle\xi_{s} ;(q-2)(\mathbf{p}, \lambda)\left|H_{\mathrm{int}}(\xi)\right| \xi_{r} ;(q-1)(\mathbf{p}, \lambda)\right\rangle \\
& \times\left\langle\xi_{r} ;(q-1)(\mathbf{p}, \lambda)\left|H_{\mathrm{int}}(\xi)\right| \xi_{0} ; q(\mathbf{p}, \lambda)\right\rangle .
\end{aligned}
$$

Following as before, substitution of Eqs. (4) and (5) into Eq. (19) further develops the above matrix element, such that

$$
\begin{aligned}
M_{\nu 0}^{(3)}(\xi)= & -q_{3}^{1 / 2} i\left(\frac{\hbar c p}{2 \varepsilon_{0} V}\right)^{3 / 2} e_{i}^{(\lambda)} e_{j}^{(\lambda)} e_{k}^{(\lambda)} \\
& \times \frac{1}{6} \sum_{r, s}\left(\left(E_{0 r}+\hbar c p\right)\left(E_{0 s}+2 \hbar c p\right)\right)^{-1} \\
& \times\left(\mu_{i}^{\nu s} \mu_{j}^{s r} \mu_{k}^{r 0}+\mu_{i}^{\nu s} \mu_{k}^{s r} \mu_{j}^{r 0}+\mu_{j}^{\nu s} \mu_{i}^{s r} \mu_{k}^{r 0}\right. \\
& \left.+\mu_{j}^{\nu s} \mu_{k}^{s r} \mu_{i}^{r 0}+\mu_{k}^{\nu s} \mu_{i}^{s r} \mu_{j}^{r 0}+\mu_{k}^{\nu s} \mu_{j}^{s r} \mu_{i}^{r 0}\right),
\end{aligned}
$$

the factor of $1 / 6$ again to offset over-counting. The above result can be recast in terms of a third rank molecular response tensor $\beta_{(i j k)}^{\nu 0}$, defined as

$$
\begin{aligned}
\beta_{(i j k)}^{\nu 0}= & \frac{1}{6} \sum_{r, s}\left[\left(E_{0 r}+\hbar c p\right)\left(E_{0 s}+2 \hbar c p\right)\right]^{-1} \\
& \times\left(\mu_{i}^{\nu s} \mu_{j}^{s r} \mu_{k}^{r 0}+\mu_{i}^{\nu s} \mu_{k}^{s r} \mu_{j}^{r 0}+\mu_{j}^{\nu s} \mu_{i}^{s r} \mu_{k}^{r 0}\right. \\
& \left.+\mu_{j}^{\nu s} \mu_{k}^{s r} \mu_{i}^{r 0}+\mu_{k}^{\nu s} \mu_{i}^{s r} \mu_{j}^{r 0}+\mu_{k}^{\nu s} \mu_{j}^{s r} \mu_{i}^{r 0}\right) .
\end{aligned}
$$

Just as for the two-photon case, the response from a polar chromophore again yields a dependence on the difference in ground- and excited-state dipoles. ${ }^{57}$

\section{Full process}

The fluorescence due to three-photon excitation can now be presented as

$$
I_{\text {flu }}^{(3)}(\phi)=\sum_{\xi} K^{(3)}\left\langle S_{(i j k) l} \bar{S}_{(m n o) p} T_{(i j k) l} \bar{T}_{(m n o) p}\right\rangle,
$$

where $K^{(3)}$ subsumes a factor $q_{3} \Rightarrow g^{(3)} \bar{q}^{3}$, conveying a cubic dependence on the input beam intensity and a linear dependence on its degree of third order coherence. ${ }^{56} \mathrm{In}$ Eq. (22), the electric polarization and molecular transition moments are described in terms of fourth rank tensors, where $S_{(i j k) l}$ and $\bar{S}_{(i j k) l}$ respectively represent $e_{i}^{(\lambda)} e_{j}^{(\lambda)} e_{k}^{(\lambda)} \bar{e}_{l}^{\left(\lambda^{\prime}\right)}$ and $\bar{e}_{i}^{(\lambda)} \bar{e}_{j}^{(\lambda)} \bar{e}_{k}^{(\lambda)} e_{l}^{\left(\lambda^{\prime}\right)}$, while $T_{(i j k) l}$ and $\bar{T}_{(i j k) l}$ correspond to $\beta_{(i j k)}^{\nu 0} \mu_{l}^{0 \alpha}$ and $\bar{\beta}_{(i j k)}^{\nu 0} \bar{\mu}_{l}^{0 \alpha}$, the final index of each again being associated with the one-photon emission. While the index $l$ and $p$ contractions in Eq. (22) would again deliver the $\cos ^{2} \theta$ factor for a rigid sample, the orientation relative to the input polarization depends on a multitude of angles, corresponding to the orientations of the transition moments $\boldsymbol{\mu}^{\nu s}, \boldsymbol{\mu}^{s r}, \boldsymbol{\mu}^{r 0}$, summed over states $r$ and $s$.

\section{ORIENTATIONAL AVERAGING}

Before the implementation of a rotational average, the general results for the fluorescence output in one-, two-, and three-photon induced systems, represented by Eqs. (9), (16), and (22), respectively, are applicable to systems in which the responsible chromophores have arbitrary orientations with respect to experimentally determined input and detection configurations. As such, these results are directly applicable to all ordered samples in which individual chromophores are held in a fixed orientation, or others comprising domains with significant local orientational correlations. To address substantially less ordered systems it is expedient to secure corresponding results for an opposite extreme-systems of completely random orientation. To this end, the above results are now subjected to an orientational averaging protocol. One-photon induced fluorescence is addressed first, highlighting procedures within the method in detail - though the simplicity of this case belies the significantly greater technical complexity in securing results for the higher order interactions. The latter calculations are extremely complex, and in the case of three-photon fluorescence they are only viable by the use of the specialized, not widely familiar techniques, as reported below. 


\section{A. One-photon induced fluorescence}

From Eq. (9), the one-photon induced fluorescence signal exhibits an implicit sum over four separate Cartesian indices, each of which can assume $x, y$, or $z$ values with respect to a chosen frame. The results are resolved through fourth-rank orientational averaging. The molecular and radiation components of the system are first uncoupled by assigning to the latter a laboratory-fixed frame of reference, denoted by Latin indices. The molecular transition moments within $T_{i j}$ and $\bar{T}_{k l}$ are similarly referred to a molecule-fixed frame, labeled by Greek indices, and the output signal is re-expressed:

$$
\begin{aligned}
I_{\mathrm{flu}}^{(1)}(\phi) & =K^{(1)}\left\langle S_{i j} \bar{S}_{k l} T_{i j} \bar{T}_{k l}\right\rangle \\
& =K^{(1)} S_{i j} \bar{S}_{k l} T_{\lambda \mu} \bar{T}_{\nu \sigma}\left\langle\ell_{i \lambda} \ell_{j \mu} \ell_{k \nu} \ell_{l \sigma}\right\rangle
\end{aligned}
$$

where the molecular and radiation reference frames are linked through the product of direction cosines between the frame axes, represented within the angular brackets. As the only parameters of Eq. (23) that are now dependent on molecular orientation, the orientational average is implemented over $\left\langle\ell_{i \lambda} \ell_{j \mu} \ell_{k v} \ell_{l \sigma}\right\rangle$, the general result for which is as follows: ${ }^{47}$

$\left\langle\ell_{i \lambda} \ell_{j \mu} \ell_{k \nu} \ell_{l \sigma}\right\rangle=\frac{1}{30}\left(\begin{array}{c}\delta_{i j} \delta_{k l} \\ \delta_{i k} \delta_{j l} \\ \delta_{i l} \delta_{j k}\end{array}\right)^{\mathrm{T}}\left(\begin{array}{ccc}4 & -1 & -1 \\ -1 & 4 & -1 \\ -1 & -1 & 4\end{array}\right)\left(\begin{array}{l}\delta_{\lambda \mu} \delta_{\nu \sigma} \\ \delta_{\lambda \nu} \delta_{\mu \sigma} \\ \delta_{\lambda \sigma} \delta_{\mu \nu}\end{array}\right)$.

The above Kronecker delta functions operate on the molecular and radiation tensors featured in Eq. (23) - for example $\delta_{i j} \delta_{k l}$ effects tensor contractions in the radiation frame, with $S_{i j} \bar{S}_{k l}$ yielding $S_{i i} \bar{S}_{k k}$. All of the ensuing results are then expressible in terms of scalar products between input and output polarization components. In the commonly utilized deployment of plane-polarized input laser light, the polarization vectors are real and the scalar product of two polarization vectors from the same beam is concisely summarized by

$$
\mathbf{e}^{(\lambda)} \cdot \mathbf{e}^{\left(\lambda^{\prime}\right)}=\delta_{\lambda \lambda^{\prime}}+\left(1-\delta_{\lambda \lambda^{\prime}}\right) \cos \phi,
$$

where $\phi$ is the angle between the input and output polarization vectors. The final result for the orientationally averaged fluorescence output emerges in terms of $\phi$ as

$$
\begin{aligned}
I_{\text {flu }}^{(1)}(\phi)= & \frac{K^{(1)}}{30}\left[\left(T_{\lambda \lambda} \bar{T}_{\mu \mu}+T_{\lambda \mu} \bar{T}_{\mu \lambda}\right)\left(3 \cos ^{2} \phi-1\right)\right. \\
& \left.-\left(2 T_{\lambda \mu} \bar{T}_{\lambda \mu}\right)\left(\cos ^{2} \phi-2\right)\right]
\end{aligned}
$$

involving three molecular invariants, $T_{\lambda \lambda} \bar{T}_{\mu \mu}, T_{\lambda \mu} \bar{T}_{\mu \lambda}$ and $T_{\lambda \mu} \bar{T}_{\lambda \mu}$-whose form and means of characterization, using linear and circular polarizations, were first identified in pioneering work by McClain. ${ }^{58,59}$ For this case of one-photon induced fluorescence, it is further possible to express the molecular tensors in Eq. (26) relative to the magnitude of the molecular transition moments $\boldsymbol{\mu}^{\nu 0}$ and $\boldsymbol{\mu}^{0 \alpha}$, and the angle between them, $\beta$, such that

$$
\begin{aligned}
I_{\mathrm{flu}}^{(1)}([\phi],[\beta])= & \frac{K^{(1)}\left|\boldsymbol{\mu}^{\nu 0}\right|^{2}\left|\boldsymbol{\mu}^{0 \alpha}\right|^{2}}{30}\left[\left(3 \cos ^{2} \phi-1\right)\right. \\
& \left.\times\left(2 \cos ^{2} \beta\right)-2\left(\cos ^{2} \phi-2\right)\right],
\end{aligned}
$$

where the identities $T_{\lambda \lambda} \bar{T}_{\mu \mu}=T_{\lambda \mu} \bar{T}_{\mu \lambda}=\left|\mu^{\nu 0}\right|^{2}\left|\mu^{0 \alpha}\right|^{2} \cos ^{2} \beta$ and $T_{\lambda \mu} \bar{T}_{\lambda \mu}=\left|\mu^{\nu 0}\right|^{2}\left|\mu^{0 \alpha}\right|^{2}$ apply. Resolving Eq. (27) for fluorescence components parallel or perpendicular to the input polarization leads to the familiar degree of fluorescence anisotropy for a randomly oriented sample. ${ }^{1,2}$ The equivalent, general results for two- and three-photon induced fluorescence, derived in the following, have not been completely determined before to our knowledge.

\section{B. Two-photon induced fluorescence}

The previously established averaging methods are now applied to Eq. (16) for the output, decoupling the molecular and radiation frames as before:

$$
I_{\text {flu }}^{(2)}(\phi)=K^{(2)} S_{(i j) k} \bar{S}_{(l m) n} T_{(\lambda \mu) \nu} \bar{T}_{(\sigma \tau) \rho}\left\langle\ell_{i \lambda} \ell_{j \mu} \ell_{k \nu} \ell_{l \sigma} \ell_{m \tau} \ell_{n \rho}\right\rangle .
$$

Delivery of the result now requires the implementation of a sixth-rank orientational average. ${ }^{47}$ It transpires that the fluorescence signal is generally expressible in terms of 15 molecular invariants (which are distinct, though generally they are not all linearly independent: ${ }^{24,60}$

$$
\begin{aligned}
& I_{\mathrm{flu}}^{(2)}(\phi)=\frac{K^{(2)}}{210}\left[\left(T_{(\lambda \lambda) \mu} \bar{T}_{(\mu \nu) \nu}+T_{(\lambda \lambda) \mu} \bar{T}_{(v \mu) \nu}+T_{(\lambda \mu) \lambda} \bar{T}_{(\mu \nu) v}+T_{(\lambda \mu) \lambda} \bar{T}_{(v \mu) \nu}+T_{(\lambda \mu) \lambda} \bar{T}_{(\nu v) \mu}+T_{(\lambda \mu) \mu} \bar{T}_{(\lambda v) v}\right.\right. \\
& \left.+T_{(\lambda \mu) v} \bar{T}_{(\lambda v) \mu}+T_{(\lambda \mu) \mu} \bar{T}_{(\nu \lambda) v}+T_{(\lambda \mu) v} \bar{T}_{(\nu \lambda) \mu}+T_{(\lambda \mu) \mu} \bar{T}_{(v v) \lambda}+T_{(\lambda \mu) v} \bar{T}_{(\mu \nu) \lambda}+T_{(\lambda \mu) v} \bar{T}_{(v \mu) \lambda}\right) \\
& \left.\times\left(3 \cos ^{2} \phi-1\right)-2\left(T_{(\lambda \lambda) \mu} \bar{T}_{(v v) \mu}+T_{(\lambda \mu) v} \bar{T}_{(\lambda \mu) v}+T_{(\lambda \mu) v} \bar{T}_{(\mu \lambda) v}\right)\left(2 \cos ^{2} \phi-3\right)\right] .
\end{aligned}
$$

Each of the above molecular invariants (the terms contracting $T$ and $\bar{T}$ tensors) is a scalar, expressing one particular aspect of the overall propensity of the chromophore to generate two-photon fluorescence. Each is expressible as a sum of four separate terms entailing specific transition moments, for example

$$
\begin{aligned}
T_{(\lambda \mu) \nu} \bar{T}_{(\lambda \mu) v}= & \alpha_{(\lambda \mu)}^{\nu 0} \mu_{\nu}^{0 \alpha} \bar{\alpha}_{(\lambda \mu)}^{\nu 0} \bar{\mu}_{\nu}^{0 \alpha} \\
= & \frac{1}{4} \sum_{r, \tilde{r}}\left[\left(E_{0 r}+\hbar c p\right)\left(E_{0 \tilde{r}}+\hbar c p\right)\right]^{-1}\left(\mu_{\lambda}^{v r} \mu_{\mu}^{r 0} \bar{\mu}_{\lambda}^{v \tilde{\mu}} \bar{\mu}_{\mu}^{\tilde{r} 0}+\mu_{\lambda}^{v r} \mu_{\mu}^{r 0} \bar{\mu}_{\mu}^{v \tilde{r}} \bar{\mu}_{\lambda}^{r 0}\right. \\
& \left.+\mu_{\mu}^{v r} \mu_{\lambda}^{r 0} \bar{\mu}_{\lambda}^{\nu \tilde{r}} \bar{\mu}_{\mu}^{\tilde{r} 0}+\mu_{\mu}^{v r} \mu_{\lambda}^{r 0} \bar{\mu}_{\mu}^{v \tilde{\mu}} \bar{\mu}_{\lambda}^{\tilde{r} 0}\right) \mu_{\nu}^{0 \alpha} \bar{\mu}_{\nu}^{0 \alpha},
\end{aligned}
$$


in which the state labels $r$ and $\tilde{r}$ identify two virtual states-which must be allowed to be different, since each appears in a separate sum. These summations preclude factorizing out the absorption and emission transition moments, without further assumptions that would compromise the generality of the result. It is, however, possible to redefine Eq. (29) in a more concise form by considering index symmetry properties, since a number of the invariants are equal, for example $T_{(\lambda \lambda) \mu} \bar{T}_{(\mu v) v}+T_{(\lambda \lambda) \mu} \bar{T}_{(v \mu) v}+T_{(\lambda \mu) \lambda} \bar{T}_{(v v) \mu}+T_{(\lambda \mu) \mu} \bar{T}_{(v v) \lambda}$ $\equiv 4 T_{(\lambda \lambda) \mu} \bar{T}_{(\mu \nu) v}$. In consequence, the 15 molecular invariants in Eq. (29) reduce to just four distinct terms:

$$
\begin{aligned}
I_{f l u}^{(2)}(\phi)= & \frac{K^{(2)}}{105}\left[\left(2 T_{(\lambda \lambda) \mu} \bar{T}_{(\mu v) v}+4 T_{(\lambda \mu) \lambda} \bar{T}_{(\mu v) v}\right)\right. \\
& \times\left(3 \cos ^{2} \phi-1\right)-\left(T_{(\lambda \lambda) \mu} \bar{T}_{(\nu v) \mu}+2 T_{(\lambda \mu) v} \bar{T}_{(\lambda \mu) v}\right) \\
& \left.\times\left(2 \cos ^{2} \phi-3\right)\right] .
\end{aligned}
$$

Despite the simple form of Eq. (31), it should be borne in mind that each invariant is in fact a sum of 27 distinct products of components of the tensor $T$ (since in each case the Cartesian indices $\lambda, \mu$, and $v$ each can take $x, y$, or $z$ values). Moreover, each of those tensor components is in general determined by combinations of transition moments that involve a tier of intermediate levels $r$, that tier being in principle of unlimited extent. The experimental determination of these individual parameters is impossible, because the above result provides for no more than two linearly independent polarization measurements. Moreover, calculational methods cannot assist; even the most sophisticated molecular software cannot usually secure the necessary convergence in the sums over states, even for relatively small molecules. However, there is sufficient information in the result Eq. (31) to yield physically meaningful interpretations, as will be shown in the discussion in Sec. IV. The value of the present method is still more evident in the following three-photon case; although the procedure for securing the result has been significantly more complex, it does in fact produce concisely expressible and tractable results.

\section{Three-photon induced fluorescence}

The orientationally averaged output signal for threephoton induced fluorescence is now considered, beginning with the re-expression of Eq. (22) as

$$
\begin{aligned}
I_{\text {flu }}^{(3)}(\phi)= & K^{(3)} S_{(i j k) l} \bar{S}_{(m n o) p} T_{(\lambda \mu v) \sigma} \bar{T}_{(\tau \rho \pi) \eta} \\
& \times\left\langle\ell_{i \lambda} \ell_{j \mu} \ell_{k \nu} \ell_{l \sigma} \ell_{m \tau} \ell_{n \rho} \ell_{o \pi} \ell_{p \eta}\right\rangle,
\end{aligned}
$$

requiring an eighth-rank average. ${ }^{61}$ In contrast to the fourth and sixth rank orientational averages already utilized, the general form of the eighth-rank expression, specifically applied as $\left\langle\ell_{i \lambda} \ell_{j \mu} \ell_{k v} \ell_{l \sigma} \ell_{m \tau} \ell_{n \rho} \ell_{\sigma \pi} \ell_{p \eta}\right\rangle$, is rarely reported owing to the extreme complexity in presenting and resolving the matrix result. However, following the same methodology, a general result has now been determined in which the three-photon induced fluorescence signal is described in terms of 105 molecular invariants. In order to present a more manageable result, it is necessary to again exploit the indexsymmetrized form of the molecular tensors, allowing the out- put signal then to be expressed much more simply in terms of just five unique molecular invariants:

$$
\begin{aligned}
I_{\text {flu }}^{(3)}(\phi)= & \frac{K^{(3)}}{315}\left[\left(3 T_{(\lambda \lambda \mu}\right)_{\mu} \bar{T}_{(\nu v \sigma) \sigma}+6 T_{(\lambda \lambda \mu) v} \bar{T}_{(\mu \nu \sigma) \sigma}\right. \\
& \left.+6 T_{(\lambda \mu \nu) \lambda} \bar{T}_{(\mu \nu \sigma) \sigma}\right)\left(3 \cos ^{2} \phi-1\right)-\left(3 T_{(\lambda \lambda \mu) v} \bar{T}_{(\mu \sigma \sigma) v}\right. \\
& \left.\left.+2 T_{(\lambda \mu \nu) \sigma} \bar{T}_{(\lambda \mu v) \sigma}\right)\left(3 \cos ^{2} \phi-4\right)\right] .
\end{aligned}
$$

Each invariant in this case comprises a sum of 81 tensor component products. As with two-photon induced fluorescence, the inherent summation over accessible intermediate states, in this case $r, \tilde{r}, s$, and $\tilde{s}$, precludes further simplification of Eq. (33).

\section{DISCUSSION}

The results of the above theory represent tools that can be applied in the analysis of polarization-determined features in two- and three-photon fluorescence from samples of considerable molecular complexity. By determining how either type of multiphoton-induced fluorescence signal responds to the orientation of a polarizer, it is in principle possible to distinguish and quantify any departure from local orientational order or disorder within a bulk sample. Key to this discrimination is the difference in angular disposition of the fluorescence polarization.

In samples whose chromophores are rigidly oriented, the fluorescence signal from an ensemble with common orientation takes the form of $\cos ^{2} \theta$ distribution with respect to the angle $\theta$ between the emission moment and the resolved polarization. On rotation of the polarizer through $180^{\circ}$ there will be an angle at which the signal is extinguished-both for singleand multi-photon induced fluorescence. However, as will be shown, the behavior from a randomly oriented sample is in general distinctively different.

First, it is interesting to observe that the equations determining multiphoton fluorescence response, namely Eqs. (31) and (33) - also Eq. (26) for the one-photon case-prove to be expressible in a relatively simple, generic form. In fact, the multiphoton fluorescence output associated with randomly disposed chromophores can be described through the following formula:

$$
\begin{aligned}
I_{\text {flu }}^{(n)}(\phi)= & K^{(n)}\left[\Lambda^{(n)}\left(3 \cos ^{2} \phi-1\right)\right. \\
& \left.-\Upsilon^{(n)}\left(n \cos ^{2} \phi-(n+1)\right)\right],
\end{aligned}
$$

with both $\Lambda^{(n)}$ and $\Upsilon^{(n)}$ representable as a sum of distinct molecular invariants, the former featuring as a coefficient of the second Legendre polynomial $\left(3 \cos ^{2} \phi-1\right)$, characteristic of time-resolved fluorescence anisotropy. There is no angle at which the $\Upsilon^{(n)}$ term can be made to vanish. However, under "magic angle" conditions where $\phi$ is $54.7^{\circ}$, the $\Lambda^{(n)}$ terms do disappear, so that the corresponding measurement should enable the identification, at least in relative terms, of $\Upsilon^{(n)}$.

To proceed with the more general case, it is helpful to cast the above expression in the form

$$
I_{\text {flu }}^{(n)}(\phi)=K^{\prime(n)}\left[n+1-y+(3 y-n) \cos ^{2} \phi\right],
$$




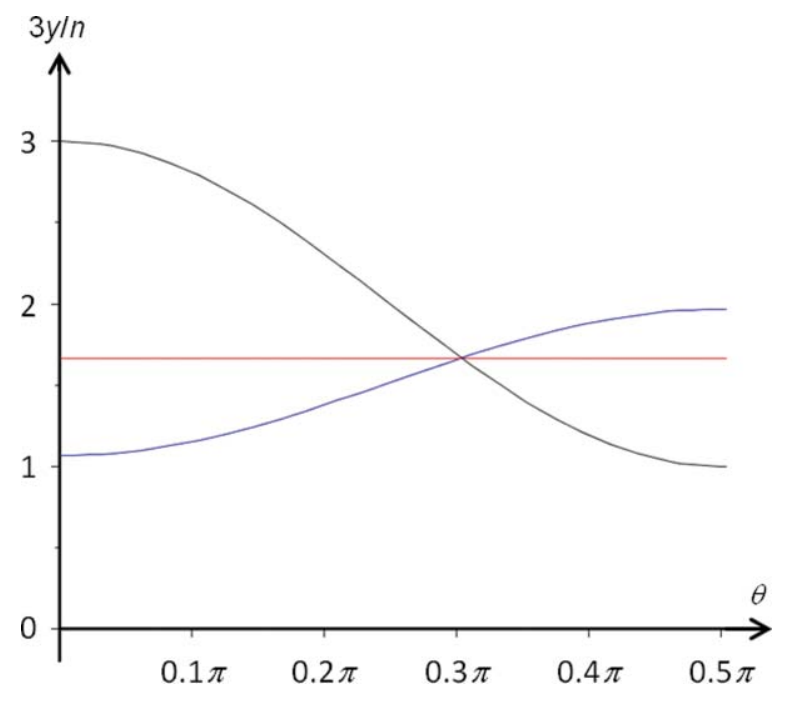

FIG. 1. Angular disposition of polarization in fluorescence produced by single-photon absorption $(n=1)$ : blue (ascending) curve $3 y / n=0.1$; red curve $3 y / n=1$; black (descending) curve $3 y / n=3$.

where $K^{\prime^{(n)}}=K^{(n)} \Upsilon^{(n)}, y=\Lambda^{(n)} / \Upsilon^{(n)}$. The latter parameter is a scalar that characterizes the relative values of the molecular invariant groupings in Eqs. (26), (31), and (33). Although the precise value of $y$ will depend on the component values of the transition tensors, it can be shown that $y$ is positive and limited to an upper bound of $(n+1)$. The graphs of Figs.1-3 exhibit the functional form of the fluorescence polarization, for one-, two-, and three-photon induced fluorescence, over the range $(0 \leq \phi \leq \pi / 2)$ - the behavior over the next quadrant being a mirror image in the ordinate axis in each case. Each graph shows the behavior for different values of $y$; the magic angle condition cited above represents the point at which the curves for all different values of $y$ intersect. These graphs appear broadly similar, though it is not in fact possible to transfer any curve to another case simply by casting against a different scale. For each type of excitation, curves

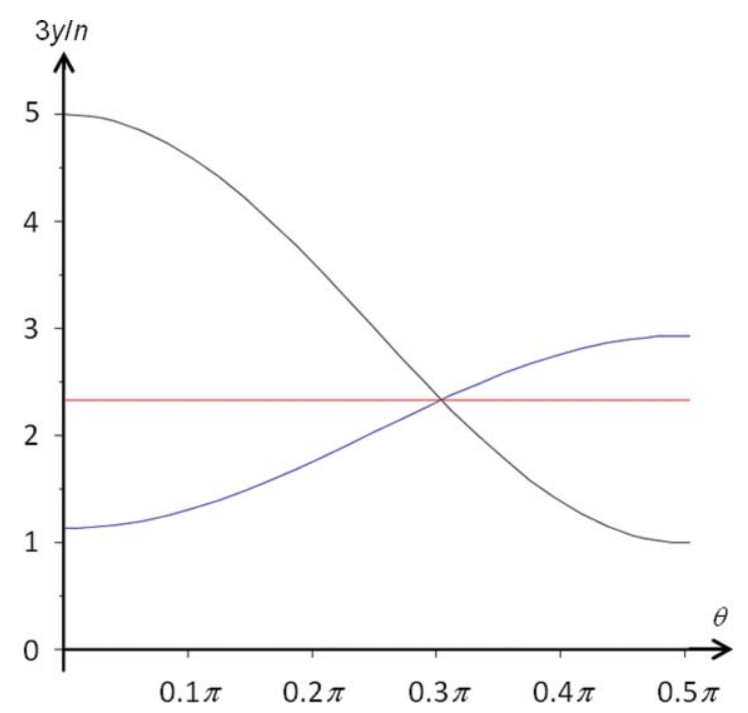

FIG. 2. Angular disposition of polarization in fluorescence produced by twophoton absorption $(n=2)$ : blue (ascending) curve $3 y / n=0.1$; red curve $3 y / n$ $=1$; black (descending) curve $3 y / n=3$. are shown for $3 y / n=0.1,1.0$, and 3.0. The curves corresponding to the case, $3 y / n=0.1$, represent an extreme condition, $\Lambda^{(n)} \ll \Upsilon^{(n)}$, characterized by strongly depolarized emission. The curves drawn for $3 y / n=1.0$ are of special interest because the fluorescence proves in each case to be independent of the resolving polarization, a general feature that has not been discovered before to our knowledge; this is a condition under which the fluorescence produced through the concerted absorption of any number of photons becomes completely unpolarized.

The results for $3 y / n=3.0$ are perhaps the most interesting, being indicative of the statistically most likely outcome. This condition arises when, within each of the relevant general Eqs. (26), (31), and (33), the featured molecular invariants are of approximately equal value-for example in the two-photon case, Eq. (31), when $T_{(\lambda \lambda) \mu} \bar{T}_{(\mu v) v}$ $=T_{(\lambda \mu) \lambda} \bar{T}_{(\mu \nu) v}=T_{(\lambda \lambda) \mu} \bar{T}_{(v v) \mu}=T_{(\lambda \mu) v} \bar{T}_{(\lambda \mu) v}$. It is remarkable that this condition leads in every case to $\Lambda^{(n)} / \Upsilon^{(n)}=y$ $=n$, a further, previously unreported result. Here there is a strong retention of polarization, the corresponding emission anisotropies $r=\left(I_{\|}-I_{\perp}\right) /\left(I_{\|}+2 I_{\perp}\right)$ proving to conform to the simple formula $r=2 n /(2 n+3)$ and yielding the following specific values: (i) $n=1, r=2 / 5=0.4$, the familiar result; $^{1}$ also (ii) $n=2, r=4 / 7=0.57$; (iii) $n=3, r=6 / 9$ $=0.67$. These limiting case results are in precise agreement with the values that arise specifically when all transition moments are considered parallel, a special case previously reported by Lakowicz et al. ${ }^{62}$ The correlation serves to verify a limiting case of the present, more general results-but it is also notable that the conditions under which such behavior arises are not only associated with parallel transition moments. The same observations will result, for example if all of the molecular transition tensor elements have similar values. It should be stressed that the above values, for physically restricted cases, cannot be regarded as absolute maxima anisotropies - even in the case of solution studies-because the approximations that lead to those results will not always hold. Although similar fluorescence anisotropies can in

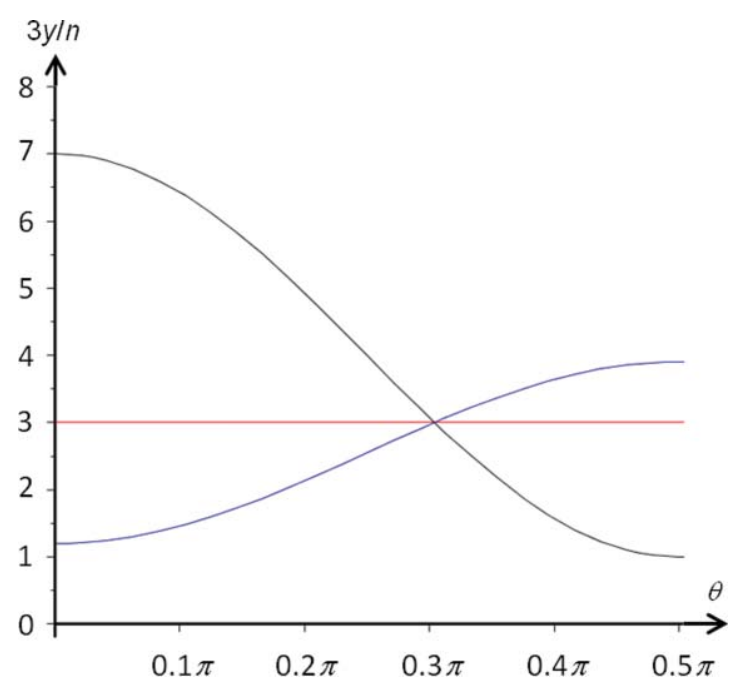

FIG. 3. Angular disposition of polarization in fluorescence produced by three-photon absorption $(n=3$ ): blue (ascending) curve $3 y / n=0.1$; red curve $3 y / n=1$; black (descending) curve $3 y / n=3$. 
principle be found even from fully oriented domains, samples of the latter kind may readily be distinguished on the basis of an anisotropy that varies with rotation of the sample itself.

In conclusion, we note that the considered cases all satisfy the condition that the ratio of maximum and minimum fluorescence intensities $I_{\min }^{(n)} / I_{\max }^{(n)}$ lies in the interval $[0,1 /(2 n+1)]$. We recall that rotation of the resolving polarizer can entirely extinguish the fluorescence from an orientationally perfectly ordered sample or domain, although the angular positions for $I_{\min }^{(n)}$ and $I_{\max }^{(n)}$ may of course not be at $\phi=$ 0 and $\pi / 2$. This suggests that in a general case the measured value of $I_{\min }^{(n)} / I_{\max }^{(n)}$ registered against the scale $[0,1 /(2 n+1)]$ should represent a robust, easily determined single-value indicator of the degree of disorder in fluorescence produced by $n$-photon excitation.

\section{ACKNOWLEDGMENTS}

This work has been funded by the award of an Engineering and Physical Sciences Research Studentship to J.M.L. We gratefully acknowledge the assistance of Matt Coles in producing the figures.

${ }^{1}$ J. R. Lakowicz, Principles of Fluorescence Spectroscopy, 2nd ed. (Kluwer Academic/Plenum, New York, 1999).

${ }^{2}$ B. Valeur, Molecular Fluorescence (Wiley-VCH, Weinheim, 2002).

${ }^{3}$ F. Festy, S. M. Ameer-Beg, T. Ng, and K. Suhling, Mol. BioSyst. 3, 381 (2007)

${ }^{4}$ J. A. Levitt, D. R. Matthews, S. M. Ameer-Beg, and K. Suhling, Curr. Opin. Biotech. 20, 28 (2009).

${ }^{5}$ C. C. Gradinaru, D. O. Marushchak, M. Samim, and U. J. Krull, Analyst 135, 452 (2010).

${ }^{6}$ S. Inoué, O. Shimomura, M. Goda, M. Shribak, and P. T. Tran, Proc. Natl. Acad. Sci. U. S. A. 99, 4272 (2002)

${ }^{7}$ S. Brasselet, V. Le Floc'h, F. Treussart, J. Roch, J. Zyss, E. Botzung-Appert, and A. Ibanez, Phys. Rev. Lett. 92, 207401 (2004).

${ }^{8}$ A. Gasecka, L. Dieu, D. Brühwiler, and S. Brasselet, J. Phys. Chem. B 114, $4192(2010)$

${ }^{9}$ B. Corry, D. Jayatilaka, B. Martinac, and P. Rigby, Biophys. J. 91, 1032 (2006).

${ }^{10}$ A. Gasecka, T. Han, C. Favard, B. R. Cho, and S. Brasselet, Biophys. J. 97, 2854 (2009).

${ }^{11}$ S. A. Empedocles, R. Neuhauser, and M. G. Bawendi, Nature (London) 399, 126 (1999)

${ }^{12}$ S. Weiss, Nat. Struct. Biol. 7, 724 (2000).

${ }^{13}$ K. D. Weston and L. S. Goldner, J. Phys. Chem. B 105, $3453(2001)$

${ }^{14}$ M. Vacha and M. Kotani, J. Chem. Phys. 118, 5279 (2003).

${ }^{15}$ A. P. Shreve, J. K. Trautman, T. G. Owens, and A. C. Albrecht, Chem. Phys. Lett. 170, 51 (1990).

${ }^{16}$ T. W. Scott, S. K. Haber, and A. C. Albrecht, J. Chem. Phys. 78, 150 (1982).

${ }^{17}$ J. R. Cable and A. C. Albrecht, J. Chem. Phys. 85, 3145 (1986).

${ }^{18}$ D. L. Andrews and B. S. Webb, J. Chem. Soc. Faraday Trans. 86, 3051 (1990).

${ }^{19}$ H. Ishitobi, Z. Sekkat, and S. Kawata, J. Opt. Soc. Am. B 23, 868 (2006).
${ }^{20}$ A. Kawski and P. Bojarski, Asian J. Spectrosc. 11, 67 (2007).

${ }^{21}$ L. Ryderfors, E. Mukhtar, and L. B.-Å. Johansson, J. Phys. Chem. A 111, 11531 (2007).

${ }^{22}$ L. Ryderfors, E. Mukhtar, and L. B.-Å. Johansson, J. Fluoresc. 17, 466 (2007).

${ }^{23}$ O. Opanasyuk, L. Ryderfors, E. Mukhtar, and L. B.-Å. Johansson, Phys. Chem. Chem. Phys. 11, 7152 (2009).

${ }^{24}$ W. M. McClain, J. Chem. Phys. 57, 2264 (1972).

${ }^{25}$ D. L. Andrews, J. Chem. Phys. 77, 2831 (1982).

${ }^{26}$ D. L. Andrews, J. Chem. Phys. 78, 1731 (1983).

${ }^{27}$ D. L. Andrews and P. J. Wilkes, J. Chem. Phys. 83, 2009 (1985).

${ }^{28}$ R. M. Williams, W. R. Zipfel, and W. W. Webb, Curr. Opin. Chem. Biol. 5, 603 (2001).

${ }^{29}$ F. Helmchen and W. Denk, Curr. Opin. Neurobiol. 12, 593 (2002).

${ }^{30}$ M. Rubart, Circ. Res. 95, 1154 (2004).

${ }^{31}$ M. Oheim, D. J. Michael, M. Geisbauer, D. Madsen, and R. H. Chow, Adv. Drug Deliver. Rev. 58, 788 (2006).

${ }^{32}$ A. Diaspro, P. Bianchini, G. Vicidomini, M. Faretta, P. Ramoino, and C. Usai, Biomed. Eng. Online 5, 36 (2006).

${ }^{33}$ J. Jhan, W. Chang, H. Chen, Y. Lee, M. Wu, C. Chen, and I. Liau, Opt. Express 16, 16431 (2008).

${ }^{34}$ P. P. Provenzano, K. W. Eliceiri, and P. J. Keely, Clin. Exp. Metastasis 26, 357 (2009).

${ }^{35}$ J. Balaji, R. Desai, and S. Maiti, Microsc. Res. Tech. 63, 67 (2004).

${ }^{36}$ S. W. Chu, S. P. Tai, C. L. Ho, C. H. Lin, and C. K. Sun, Microsc. Res. Tech. 66, 193 (2005).

${ }^{37}$ D. L. Andrews and W. A. Ghoul, J. Chem. Phys. 75, 530 (1981).

${ }^{38}$ V. Galasso, J. Chem. Phys. 92, 2495 (1990).

${ }^{39}$ A. Pasquarello and A. Quattropani, Phys. Rev. B 43, 3837 (1991).

${ }^{40}$ V. Galasso, Chem. Phys. 161, 189 (1992).

${ }^{41}$ B. N. Jagatap and W. J. Meath, J. Opt. Soc. B 19, 2673 (2002).

${ }^{42}$ E. A. Power, Introductory Quantum Electrodynamics (Longmans, London, 1964).

${ }^{43}$ W. P. Healy, Non-Relativistic Quantum Electrodynamics (Academic, London, 1982).

${ }^{44}$ D. P. Craig and T. Thirunamachandran, Molecular Quantum Electrodynamics (Dover, New York, 1998).

${ }^{45}$ R. G. Woolley, in Handbook of Molecular Physics and Quantum Chemistry, edited by S. Wilson (Wiley, Chichester, 2003), pp. 657.

${ }^{46}$ A. Salam, Int. Rev. Phys. Chem. 27, 405 (2008).

${ }^{47}$ D. L. Andrews and T. Thirunamachandran, J. Chem. Phys. 67, 5026 (1977).

${ }^{48}$ G. D. Scholes, J. Chem. Phys. 121, 10104 (2004).

${ }^{49}$ V. M. Huxter and G. D. Scholes, J. Chem. Phys. 132, 104506 (2010).

${ }^{50}$ A. Salam, J. Phys. B 39, S651 (2006).

${ }^{51}$ A. Salam, J. Phys.: Conf. Ser. 161, 012040 (2009).

${ }^{52}$ E. Collini and G. D. Scholes, J. Phys. Chem. A 113, 4223 (2009).

${ }^{53}$ F. Šanda, V. Perlík, and S. Mukamel, J. Chem. Phys. 133, 014102 (2010).

${ }^{54}$ J. M. Leeder, D. S. Bradshaw, and D. L. Andrews, "Laser-Controlled Fluorescence in two-level systems," J. Phys. Chem. B (in press).

${ }^{55} \mathrm{~L}$. Mandel and E. Wolf, Optical Coherence and Quantum Optics (Cambridge University Press, Cambridge, 1995).

${ }^{56} \mathrm{R}$. Loudon, The Quantum Theory of Light, 3rd ed. (Oxford University Press, Oxford, 2000).

${ }^{57}$ W. J. Meath and E. A. Power, J. Phys. B 17, 763 (1984).

${ }^{58}$ W. M. McClain, J. Chem. Phys. 55, 2789 (1971).

${ }^{59}$ P. R. Monson and W. M. McClain, J. Chem. Phys. 56, 4817 (1972).

${ }^{60}$ W. M. McClain, J. Chem. Phys. 58, 314 (1973).

${ }^{61}$ D. L. Andrews and W. A. Ghoul, J. Phys. A 14, 1281 (1981).

${ }^{62}$ J. R. Lakowicz, I. Gryczynski, H. Malak, M. Schrader, P. Engelhardt, H. Kano, and S. W. Hell, Biophys. J. 72, 567 (1997). 